\title{
Gd $^{3+}$-Doped Magnetic Nanoparticles for Biomedical Applications
}

\author{
A. P. Budnyk, ${ }^{1}$ T. A. Lastovina, ${ }^{1}$ A. L. Bugaev ${ }^{D},{ }^{1}$ V. A. Polyakov, ${ }^{1}$ K. S. Vetlitsyna-Novikova, ${ }^{1}$ \\ M. A. Sirota, ${ }^{2,3}$ K. G. Abdulvakhidov, ${ }^{1}$ A. G. Fedorenko, ${ }^{4}$ E. O. Podlesnaya, ${ }^{5}$ \\ and A. V. Soldatov $\mathbb{D}^{1}$
}

\author{
${ }^{1}$ The Smart Materials Research Center, Southern Federal University, 344090 Rostov-on-Don, Russia \\ ${ }^{2}$ Don State Technical University, 344010 Rostov-on-Don, Russia \\ ${ }^{3}$ Department of Physics, Southern Federal University, 344090 Rostov-on-Don, Russia \\ ${ }^{4}$ Modern Microscopy Center, Academy of Biology and Biotechnology, Southern Federal University, \\ 344090 Rostov-on-Don, Russia \\ ${ }^{5}$ Department of Chemistry, Southern Federal University, 344090 Rostov-on-Don, Russia
}

Correspondence should be addressed to A. L. Bugaev; abugaev@sfedu.ru

Received 27 February 2018; Accepted 11 June 2018; Published 2 August 2018

Academic Editor: K. S. V. Krishna Rao

Copyright (c) 2018 A. P. Budnyk et al. This is an open access article distributed under the Creative Commons Attribution License, which permits unrestricted use, distribution, and reproduction in any medium, provided the original work is properly cited.

\begin{abstract}
Magnetic nanoparticles (MNPs) made of iron oxides with cubic symmetry $\left(\mathrm{Fe}_{3} \mathrm{O}_{4}, \gamma-\mathrm{Fe}_{2} \mathrm{O}_{3}\right)$ are demanded objects for multipurpose in biomedical applications as contrast agents for magnetic resonance imaging, magnetically driven carriers for drug delivery, and heaters in hyperthermia cancer treatment. An optimum balance between the right particle size and good magnetic response can be reached by a selection of a synthesis method and by doping with rare earth elements. Here, we present a microwave-assisted polyol synthesis of iron oxide MNPs with actual gadolinium (III) doping from 0.5 to 5.1 mol.\%. The resulting MNPs have an average size of $14 \mathrm{~nm}$ with narrow size distribution. Their surface was covered by a glycol layer, which prevents aggregation and improves biocompatibility. The magnetic hyperthermia test was performed on $1 \mathrm{and} 2 \mathrm{mg} / \mathrm{ml}$ aqueous colloidal solutions of MNPs and demonstrated their ability to rise the temperature by $3^{\circ} \mathrm{C}$ during a $20-30 \mathrm{~min}$ run. Therefore, the obtained $\mathrm{Gd}^{3+} \mathrm{MNPs}$ are the promising material for biomedicine.
\end{abstract}

\section{Introduction}

Magnetic nanoparticles (MNPs) have been attracting special attention of researchers in recent years due to their unique properties such as size-dependent magnetic behavior (including large magnetic moment and superparamagnetism), low toxicity for living organisms, and possession of active surface for the adsorption/immobilization of ligands and other metals [1]. They have possible applications in catalysis $[2,3]$, biomedicine [4], water purification from heavy metals $[5,7]$, magnetic data recording devices [8], and so on. MNPs can be obtained by a variety of wet chemical methods, including a sol-gel method with the use of $\mathrm{NaCl}$ and $\mathrm{NaH}_{2} \mathrm{PO}_{4}$ to control initial aggregation [9], a hydrothermal synthesis with polyethyleneimine as a capping ligand [10], and a solvothermal synthesis in ethylene glycol (EG) with oleylamine and 1,3diaminopropane as surfactants and sodium acetate as a steric stabilizer [11]. In particular, the use of glycols in the synthesis allows producing MNPs with increased hydrophilicity and improved biocompatibility.

Among magnetic materials for nano-biomedicine, magnetite $\left(\mathrm{Fe}_{3} \mathrm{O}_{4}\right)$ and maghemite $\left(\gamma-\mathrm{Fe}_{2} \mathrm{O}_{3}\right)$ are the most widely used. Both oxides are ferrimagnetic; however, in a nanoconfined form, they exhibit superparamagnetic behavior, where magnetic susceptibility is higher than that in paramagnetic materials [12]. Magnetite is a preferred material for biomedical applications due to a larger magnetic moment and availability of relatively simple synthesis methods. It has been reportedly used in magnetic cell separation [13], magnetocytolysis [14], magnetic hyperthermia [15], tracking of 
biological components [16], targeting and controlled release of drugs [17], and contrast agents in magnetic resonance imaging (MRI) [18].

Introduction of the rare earth elements, such as samarium (III), europium (III), and gadolinium (III) ions, into the structure of MNPs improve their magnetic and optical properties. This task is not trivial as the size of lanthanides is too large to let them be easily incorporated into the crystalline lattice of iron oxide [19]. Auffan et al. [5, 7] discussing a guest metal adsorption at the surface of MNPs smaller than $20 \mathrm{~nm}$ considered an oxidative transformation of freshly synthesized MNPs from magnetite to maghemite by desorption of $\mathrm{Fe}^{2+}$ ions and creation of vacancies in the octahedral sublattice. The resistance to oxidation of magnetite MNPs may be improved by substitution of the $\mathrm{Fe}^{3+}$ ions with doping rare earth elements like $\mathrm{Sm}^{3+}$ and $\mathrm{Eu}^{3+}$ [20]. Huan et al. [21] studied the impact of the doping with $\mathrm{Eu}^{3+}\left(0.7-18.9\right.$ mol.\%) and $\mathrm{Sm}^{3+}$ (1.9-13.2 mol.\%) ions on the structure and magnetic and photoluminescence properties of magnetite MNPs. It was concluded that magnetic saturation of the doped material depends on the quantity of added lanthanide, that selectively substitutes $\mathrm{Fe}^{3+}$ ions in the octahedral and tetrahedral sublattices (at high quantities of the dopant) of the spinel phase. To obtain the samples of MNPs, the authors carried out a solvothermal synthesis at $180^{\circ} \mathrm{C}$ for $6 \mathrm{~h}$ using $\mathrm{FeCl}_{3} \cdot 6 \mathrm{H}_{2} \mathrm{O}$ and anhydrous $\mathrm{EuCl}_{3}$ and $\mathrm{SmCl}_{3}$ as metal precursors and $\mathrm{EG}$ as a reducing agent. Much less has been communicated on doping of magnetite MNPs with $\mathrm{Gd}^{3+}$ ions. For instance, Choi et al. [22] investigated the effect of gadolinium doping on water proton relaxivities. Ultrasmall MNPs with actual gadolinium content of 0.2 and $0.4 \mathrm{~mol} . \%$ were obtained by processing the mixture of $\mathrm{GdCl}_{3} \cdot x \mathrm{H}_{2} \mathrm{O}, \mathrm{FeCl}_{3} \cdot 6 \mathrm{H}_{2} \mathrm{O}$, and triethylene glycol at $250^{\circ} \mathrm{C}$ for $24 \mathrm{~h}$.

We have recently reported on the preparation of MNPs doped with rare earth elements [23-25]. In particular, we obtained the $\mathrm{Sm}^{3+}$-doped (4-9wt.\%) $\mathrm{Fe}_{3} \mathrm{O}_{4}$ MNPs with an average size falling in the $6-18 \mathrm{~nm}$ interval (depending on the EG/PEG ratio) by a polyol-mediated microwave-assisted (MW) synthesis [23] or those adjusted to $9 \mathrm{~nm}$ (when adding a capping agent) by a polyol-mediated solvothermal synthesis [24]. In another study, we obtained $\mathrm{Gd}^{3+}$-doped (4.2 wt.\%) $\gamma-\mathrm{Fe}_{2} \mathrm{O}_{3}$ MNPs of $12 \mathrm{~nm}$ by a coprecipitation method [25]. Since the quantity of a rare earth dopant is of paramount importance for defining the MNPs response to an external magnetic field, we performed a systematic study on $\mathrm{Gd}^{3+}$-doped MNPs with an initial loading of 1-9 mol.\%. The MNPs were prepared by a polyol MW method, exploiting the benefits of reduced reaction time and the homogenous heat supply into the reaction mixture [26]. Unexpectedly, the MW method has been scarcely used up to date for the preparation of iron oxides doped with lanthanides. The obtained materials were characterized by various techniques to establish their structural properties and were employed for a hyperthermia test, confirming their good relevance for biomedical applications.

\section{Materials and Methods}

2.1. Synthesis of MNPs. The reagents were purchased from commercial suppliers (Alfa Aesar and Sigma-Aldrich) and used as such. The initial molar fraction of gadolinium ions in respect to total amount of iron and gadolinium ions was varied from 0.5 to 9 mol.\%. In a typical synthesis (Table 1 for the whole set of samples), $0.665 \mathrm{~g}$ of anhydrous iron (III) chloride and $0.001 \mathrm{~g}$ of gadolinium (III) chloride were dissolved in $10 \mathrm{ml}$ of triethylene glycol. A solution of $0.096 \mathrm{~g}$ of sodium hydroxide in $7.4 \mathrm{ml}$ of triethylene glycol was prepared apart. All solutions were mixed at room temperature and then continuously stirred under flowing argon for $30 \mathrm{~min}$. The reaction mixture was placed in a $\mathrm{MW}$ reactor CEM Discover SP $(175 \mathrm{~W})$ and kept at $220^{\circ} \mathrm{C}$ for $1.5 \mathrm{~h}$ with a medium rate stirring. The fine black-colored colloidal MNPs were formed in the reaction mixture. The mixture was cooled to room temperature, and the particles were collected with a permanent magnet. They were washed 5 times with distilled water and once with ethyl alcohol and then dried in vacuum at $40^{\circ} \mathrm{C}$ for $12 \mathrm{~h}$. The samples were labeled according to the actual gadolinium content as shown in Table 1.

2.2. Characterization Techniques. Samples were examined on a Bruker D2 Phaser X-ray diffractometer $\left(\mathrm{Cu} \mathrm{K}_{\alpha}, \lambda=\right.$ $1.5417 \AA$ ) with a step of $0.02^{\circ}$. X-ray diffraction (XRD) patterns were analyzed by Jana2006 program [27], following the procedure elsewhere $[28,29]$. X-ray fluorescence (XRF) analysis was carried out on a two-dimensional micro-X-ray fluorescence spectrometer Bruker M4 Tornado. The whole set of samples was examined on a transmission electron microscope (TEM) FEI Tecnai G2 Spirit BioTWIN. At least 400 particles were analyzed for every sample to build a particle size distribution histogram. High-resolution TEM (HR-TEM) and scanning TEM (STEM), selected area electron diffraction (SAED), and energy dispersive spectroscopy (EDS) imaging were performed on FEI Tecnai G2 F20S-TWIN. Infrared (IR) spectra were measured on a Fourier-transform infrared (FTIR) Bruker Vertex 70 spectrometer in transmittance with 64 scans per spectrum of $2 \mathrm{~cm}^{-1}$ resolution, pelleting the samples with $\mathrm{KBr}$ in $1: 150$ weight ratio. Spectra were converted in the absorbance values and baseline corrected (by a standard rubberband correction) using the instrument's Opus software. Calorimetric measurements for calculation of specific absorption rate (SAR) were done on a LocalHyperThermLabUnit TOR 04/16 experimental setup (Nanomaterials LLC, Russia) at $218.9 \mathrm{kHz}$ with a magnetic field flux of $20 \mathrm{mT}$ (15923 Oe).

\section{Results and Discussion}

3.1. Crystalline Phase and Actual $G d^{3+}$ Content of MNPs. Since gadolinium doping of iron oxides proceeds inefficiently due to a large difference in ionic radii of $\mathrm{Gd}^{3+}$ and $\mathrm{Fe}^{3+}$, the first thing to control after the synthesis was the actual quantity of the dopant in the samples. The values were processed from XRF data and presented in Table 1 together with respective initial loading of gadolinium. As it comes from comparison of Table 1, the actual content of $\mathrm{Gd}^{3+}$ ions in the resulting material is significantly lower than the initially loaded one. This is an undesirable fact, but similar situation was already observed by other authors reporting on 
TABLE 1: Properties of synthesized MNPs: initial loading $\left(\chi_{\text {ini }}\right)$ and actual content $\left(\chi_{\text {act }}\right.$, by XRF data) of Gd ${ }^{3+}$ ions in the samples, a cubic lattice parameter $(a)$, and average crystallite size of MNPs $\left(d_{\mathrm{av}}\right)$ calculated from XRD and TEM data, and SAR values evaluated after the hyperthermia test.

\begin{tabular}{|c|c|c|c|c|c|c|c|}
\hline Sample name & $\begin{array}{c}\chi_{\mathrm{ini}} \mathrm{Gd}^{3+} \\
(\mathrm{mol} . \%)\end{array}$ & $\begin{array}{c}\chi_{\text {act }} \mathrm{Gd}^{3+} \\
(\mathrm{mol} \%)\end{array}$ & $\begin{array}{l}\text { Lattice } \\
\text { system }\end{array}$ & $\begin{array}{c}a^{(\mathrm{XRD})} \\
(\AA)\end{array}$ & $\begin{array}{c}d_{\mathrm{av}}(\mathrm{XRD}) \\
(\mathrm{nm})\end{array}$ & $\begin{array}{c}d_{\mathrm{av}} \text { (TEM) } \\
(\mathrm{nm})\end{array}$ & SAR (W/g) \\
\hline MNP-Gd0\% & 0 & 0 & Cubic $^{\S}$ & $8.368(8)$ & 6 & 6.6 & 15.6 \\
\hline $\begin{array}{l}\text { MNP- } \\
\text { Gd0.5\% }\end{array}$ & 1 & 0.5 & Cubic $^{\mathfrak{S}}$ & $8.385(2)$ & 10 & 14.9 & 12.5 \\
\hline $\begin{array}{l}\text { MNP- } \\
\text { Gd0.6\% }\end{array}$ & 2.7 & 0.6 & Cubic $^{\mathfrak{S}}$ & $8.348(3)$ & 15 & 15.5 & 15.9 \\
\hline $\begin{array}{l}\text { MNP- } \\
\text { Gd2.5\% }\end{array}$ & 5.6 & 2.5 & $\begin{array}{l}\text { Cubic } \\
+ \text { orth. }^{*}\end{array}$ & $8.366(5)$ & 14 & 14.1 & 15.5 \\
\hline $\begin{array}{l}\text { MNP- } \\
\text { Gd5.1\% }\end{array}$ & 7 & 5.1 & Cubic $^{\S}$ & $8.386(1)$ & 15 & 13.3 & 18.0 \\
\hline $\begin{array}{l}\text { MNP- } \\
\text { Gd4.3\% }\end{array}$ & 9 & 4.3 & $\begin{array}{l}\text { Cubic } \\
\text { + }^{\text {orth. }}{ }^{\dagger}\end{array}$ & - & - & 8.5 & 13.1 \\
\hline
\end{tabular}

${ }^{\varsigma}$ Pure inverse spinel phase $\left(\mathrm{Fe}_{3} \mathrm{O}_{4}\right.$ or/and $\left.\gamma-\mathrm{Fe}_{2} \mathrm{O}_{3}\right)$; ${ }^{*}$ orthorhombic goethite $(\alpha-\mathrm{FeOOH}$ with $a=4.59(1) \AA, b=9.90(5) \AA$, and $c=3.00(1) \AA)$ and spinel phase † orthorhombic goethite $(\alpha$-FeOOH with $a=4.590(3) \AA, b=9.935(8) \AA$, and $c=3.025(2) \AA)$ and spinel phase.

iron oxides doping with rare earth elements [18]. Let us briefly discuss the possible reasons without entering into matters of detail. The aforementioned difficulty for the larger $\mathrm{Gd}^{3+}$ ion to accommodate in the iron oxide lattice implies a fraction of gadolinium being preserved in the reaction mixture in either a form of unreacted salt or, more likely, as a hydroxide $\left(\mathrm{Gd}(\mathrm{OH})_{3}\right)$. At the end of synthesis, it would not be recovered with a magnet and will not remain in the final material. Since $\mathrm{Gd}^{3+}$ ion preferentially occupies the octahedral sites of the inverse spinel structure of $\mathrm{Fe}_{3} \mathrm{O}_{4}$ while hardly entering the smaller tetrahedral sites, only a certain relatively high concentration of gadolinium will be needed to set the conditions for its incorporation into the crystal lattice in a greater extent.

Figure 1 presents the XRD patterns of the synthesized iron oxides with a different $\mathrm{Gd}^{3+}$ content as well as that of pristine iron oxide from the same synthesis (patterns are vertically shifted for clarity). Most of the patterns (excluding those for the samples with gadolinium doping of 2.5 and 4.3 mol.\%) contain the same sequence of the reflections at $2 \theta=30.2,35.5,43.2,53.5,57.2$, and $62.7^{\circ}$ indicative for an inverse spinel phase and corresponding to (220), (311), (400), (422), (511), and (440) crystallographic planes, respectively, which is immanent to both magnetite and maghemite [1]. There are no other reflections in these patterns, which evidences the phase purity of the samples. This would mean that gadolinium is evenly dispersed in the lattice without forming a distinct phase. Both MNP-Gd2.5\% and MNP-Gd4.3\% samples contain an additional orthorhombic phase, which was assigned to goethite $(\alpha-\mathrm{FeOOH})$. The established pure phases and their mixtures are summarized in Table 1 in terms of cubic and orthorhombic lattice systems.

We noted that formation of the iron hydroxide phase occurs at relatively high $\mathrm{Gd}^{3+}$ loading. This might be a result of excessive presence of the $\mathrm{Fe}^{3+}$ ions in the reaction mixture due to favorable incorporation of $\mathrm{Gd}^{3+}$ ions into the growing particles. We will illustrate this assumption by equations of chemical reactions taking place during the synthesis of MNPs.

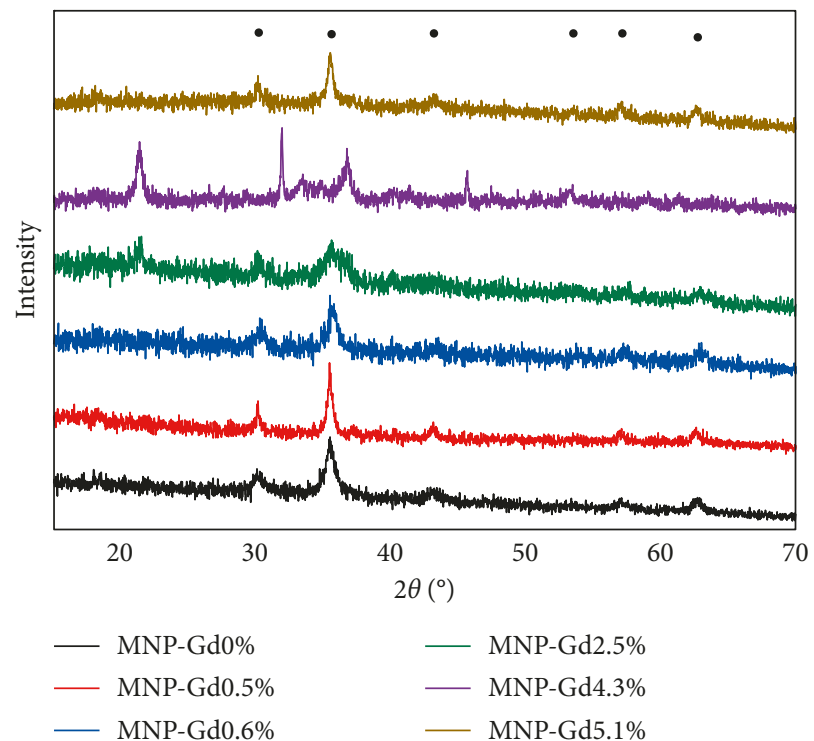

Figure 1: XRD patterns of (1) MNP-Gd0\%, (2) MNP-Gd0.5\%, (3) MNP-Gd0.6\%, (4) MNP-Gd2.5\%, (5) MNP-Gd4.3\%, and (6) MNPGd5.1\%. The solid dots indicate the diffractions of the pure magnetite phase (JCPDS 01-074-1910).

The magnetite MNPs are formed in a coprecipitation reaction:

$$
2 \mathrm{Fe}^{3+}+\mathrm{Fe}^{2+}+8 \mathrm{OH}^{-} \longrightarrow \mathrm{Fe}_{3} \mathrm{O}_{4}+4 \mathrm{H}_{2} \mathrm{O}
$$

Presence of the $\mathrm{Fe}^{2+}$ ions can be explained by the partial reduction of the $\mathrm{Fe}^{3+}$ ions from the iron precursor according to the following reactions [24]:

$$
\begin{aligned}
\mathrm{CH}_{2} \mathrm{OH}-\mathrm{CH}_{2} \mathrm{OH} \longrightarrow & \mathrm{CH}_{3} \mathrm{CHO}+\mathrm{H}_{2} \mathrm{O} \\
2 \mathrm{Fe}^{3+}+2 \mathrm{CH}_{3} \mathrm{CHO}+\mathrm{OH}^{-} \longrightarrow & 2 \mathrm{Fe}^{2+}+\mathrm{CH}_{3} \mathrm{CO} \\
& -\mathrm{COCH}_{3}+2 \mathrm{H}_{2} \mathrm{O}
\end{aligned}
$$

The excess of $\mathrm{Fe}^{3+}$ ions (that does not participate in formation of MNPs due to the presence of competitive $\mathrm{Gd}^{3+}$ ions) can react with abundant hydroxide ions: 


$$
\mathrm{Fe}^{3+}+3 \mathrm{OH}^{-} \longrightarrow \mathrm{Fe}(\mathrm{OH})_{3}
$$

Then, iron (III) hydroxide can form goethite by partial dehydration [30]:

$$
\mathrm{Fe}(\mathrm{OH})_{3} \longleftrightarrow \mathrm{FeO}(\mathrm{OH})+\mathrm{H}_{2} \mathrm{O}
$$

It is unlikely for the $\mathrm{Gd}^{3+}$ ions to enter the growing crystalline lattice of the goethite phase. Such sample will combine NPs of two phases, what we have actually seen from XRD data. This also explains why the lower gadolinium content was detected in the resulting sample after initial loading of $9 \mathrm{~mol}$ \% compared to that of $7 \mathrm{~mol} . \%$.

For the MNPs of a spinel phase, we have calculated the lattice parameter and the average crystallite size (by Debye-Scherrer equation). The respective values are presented in Table 1.

\subsection{MNPs Size, Morphology, and Elements Distribution.}

The results of TEM measurements are presented in Figure 2 together with respective particle size distribution histograms. All $\mathrm{Gd}^{3+}$-doped samples (except MNP-Gd5.1\%) consist of both spherical (smaller) and polyhedral (bigger) particles with sizes in the range from 6 to $24 \mathrm{~nm}$. Particle size distribution is narrow, and its profile is close to normal one for the most cases. Both MNP-Gd4.3\% and MNP-Gd0\% samples are characterized by more tight distribution interval (from 6 to $10 \mathrm{~nm}$ ). The weighted average values of MNPs size for each sample were calculated from the obtained distributions and presented in Table 1. It can be seen that regardless of the gadolinium content the average size of MNPs is $14 \mathrm{~nm}$ (excluding the MNP-Gd4.3\% sample). This observation implies that the adopted synthesis method shows a good reproducibility in terms of particle size, and its parameters (such as time, duration, and concentration of metal precursors in reaction volume) would define the main dimension of the resulting MNPs.

The sample with relatively high gadolinium content, MNP-Gd5.1\%, was chosen for the studies by applying the advanced electron microscopy techniques (Figure 2). The HR-TEM images (Figures 3(a) and 3(b)) confirmed a polyhedral shape of MNPs. The obtained SAED images (Figure 3(c)) allow calculating the values of interplanar distances as 2.92 and $2.52 \AA$, which correspond to (311) and (220) planes of the $\mathrm{Fe}_{3} \mathrm{O}_{4}$ cubic phase, respectively. The measured interplanar spacing is $0.294 \mathrm{~nm}$, evidencing the (220) plane of the cubic structure of magnetite. The combined STEM-EDS mapping (Figures 3(d) and 3(e)) on the same region of the sample evidences that iron and gadolinium are uniformly distributed across the volume of MNPs. As presented at the EDS histogram (Figure 3(f)), the total weight content of gadolinium and iron in a given point of the MNP-Gd5.1\% sample can be estimated as 6.8 and $71.86 \%$, respectively. Such weight ratio corresponds to $3.3 \mathrm{~mol} . \%$ of gadolinium. This value is 1.5 times lower than that estimated from the XRF data. Such difference is often happening when two dissimilar techniques are used.
3.3. MNPs Vibrational Profile and Surface Coverage. FTIR spectra of the synthesized samples are shown in Figure 4(a) (vertically shifted for clarity). The dominating complex band below $700 \mathrm{~cm}^{-1}$ is due to MNPs stretching modes. At first sight, its complex profile does not change appreciably with increasing Gd content. Instead, when normalized at maximum intensity (Figure 4(b)), the band's shape changes with gadolinium content, most notably at about $630 \mathrm{~cm}^{-1}$. The profile of this band can be fitted with tree lorentzians (Figure 4(b)) with maxima falling at 571, 630, and $700 \mathrm{~cm}^{-1}$. The first frequency is a typical $\nu \mathrm{Fe}-\mathrm{O}$ vibration in magnetite [31]. That is one of the two characteristic bands of the $\mathrm{Fe}_{3} \mathrm{O}_{4}$ phase at $570 \mathrm{~cm}^{-1}\left(\nu_{1}\right)$ and at $390 \mathrm{~cm}^{-1}\left(\nu_{2}\right)$ [32]. They originate from the $\mathrm{Fe}-\mathrm{O}$ stretching mode of the tetrahedral and octahedral sites for the $v_{1}$ frequency band and the $\mathrm{Fe}-\mathrm{O}$ stretching mode of the octahedral sites for the $v_{2}$ frequency band. Introduction of defects into the structure causes widening and eventual splitting of both $\nu_{1}$ and $\nu_{2}$ bands, giving the rise to new IR bands at around 430 and $630 \mathrm{~cm}^{-1}$. The $\gamma-\mathrm{Fe}_{2} \mathrm{O}_{3}$ phase is considered as a defective form of $\mathrm{Fe}_{3} \mathrm{O}_{4}$, and it has characteristic bands at 700 and $630 \mathrm{~cm}^{-1}$ [33]. Turning to our samples, we found that the component at $630 \mathrm{~cm}^{-1}$ exhibits a slight growth with increasing gadolinium content (Figure 4(b)), supporting an assumption about introduction of $\mathrm{Gd}^{3+}$ ions into the $\mathrm{Fe}_{2} \mathrm{O}_{3}$ sublattice of the $\mathrm{Fe}_{3} \mathrm{O}_{4}$ crystal phase of MNPs. Similar trend can be found when considering evaluation of the shape in a complimentary three-component band at lower frequencies. For the sake of clarity, we report its enlarged profile and fitting results for just two samples of MNP-Gd0\% and MNPGd5.1\% (Figure 4(c)). By comparing the relative intensities of fitting components, the rise of the band at $440 \mathrm{~cm}^{-1}$ with increasing gadolinium content is clearly evident.

Another iron compound, whose presence has been assumed in the samples based on the XRD data, is goethite, which expresses the $-\mathrm{OH}$ stretch vibrations at 1124,890 , and $810 \mathrm{~cm}^{-1}$ [33]. These frequencies comply well with the bands clearly visible for the MNP-Gd2.5\% and MNP-Gd4.3\% samples.

The spectroscopic features in the adjacent $1600-800 \mathrm{~cm}^{-1}$ interval contain a vibrational signature of glycol [34] which was involved in the synthesis and was preserved in the samples. Its spectral fingerprint is easily seen in spectrum of the MNP-d2.5\% sample. In brief, two groups of bands at about 1100 and $900 \mathrm{~cm}^{-1}$ originate from out-of-plane and in-plane $\gamma \mathrm{CH}_{2}$ vibrations (bands at 1118 and $935 \mathrm{~cm}^{-1}$ ) and stretching modes of skeleton $\mathrm{C}-\mathrm{O}$ bonds (bands at 1070 and $890 \mathrm{~cm}^{-1}$ ), respectively. A spectral envelope at about $1400 \mathrm{~cm}^{-1}$ is composed of various bending modes of $\mathrm{CH}_{2}$ groups.

The distinct band at $1600 \mathrm{~cm}^{-1}$ (see MNP-Gd0\% in Figure 4) appears after the functionalization of glycol onto the surface of magnetite NPs and will be commented in more detail. The high-frequency side of the band is formed by contribution from the $\delta \mathrm{OH}$ vibration of water moieties falling at $\sim 1630 \mathrm{~cm}^{-1}$. The right side of the band contains the strong $\nu \mathrm{C}-\mathrm{O}$ vibration, which appears due to a covalent bonding between iron and carbonyl oxygen of glycol [35]. 

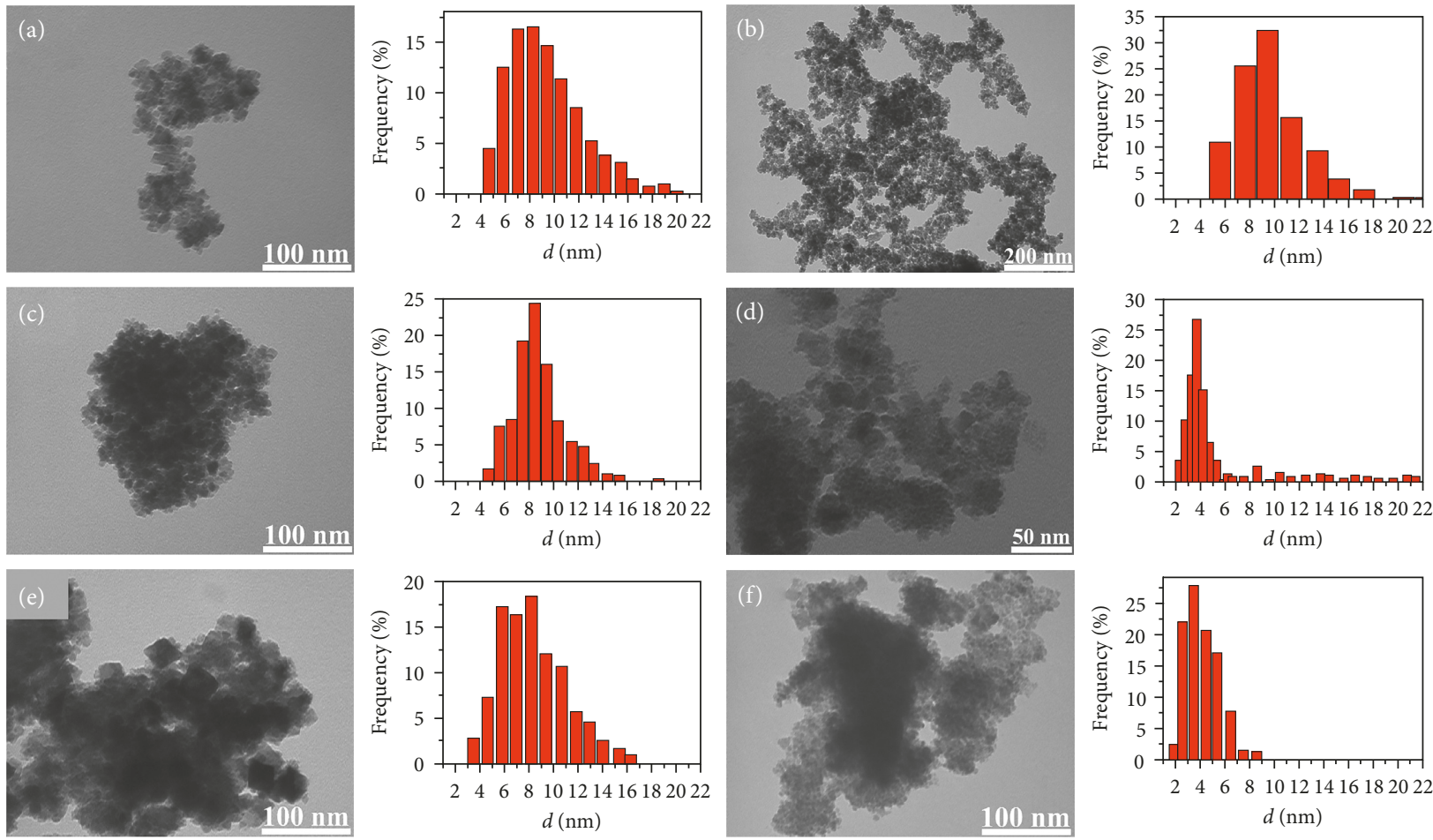

FiguRE 2: TEM images and respective particle size distribution histograms for (a) MNP-Gd0.5\%, (b) MNP-Gd0.6\%, (c) MNP-Gd2.5\%, (d) MNP-Gd4.3\%, (e) MNP-Gd5.1\%, and (f) MNP-Gd0\% samples.

Taking into account a featureless neighboring band at $1400 \mathrm{~cm}^{-1}$, we may assume of being in presence of carboxyl's oxygen bonding in unidentate arrangement [13] due to a large separation $\left(\Delta \sim 200 \mathrm{~cm}^{-1}\right)$ between the asymmetric $v_{\text {as }}\left(\mathrm{COO}^{-}\right)$and symmetric $v_{s}\left(\mathrm{COO}^{-}\right)$vibrations. With increasing amount of added $\mathrm{Gd}^{3+}$ ions, this separation decreases ( $\Delta=135 \mathrm{~cm}^{-1}$ for the MNP-Gd2.5\% sample), evidencing a chelating arrangement of the glycol ligands on the surface of MNPs.

Although stretching modes of hydroxyl groups in glycol fall in the $3700-3600 \mathrm{~cm}^{-1}$ interval, they are hidden by stretching vibrations of water molecules physisorbed on potassium bromide (used for the pelleting), leading to formation of a very broadband in the $3600-3000 \mathrm{~cm}^{-1}$ range. Nearby, there is a complex band at about $2850 \mathrm{~cm}^{-1}$ due to the overlapping asymmetric and symmetric stretching vibrations of methylene groups.

3.4. Hyperthermia Test with MNPs. As it is known, magnetic induction hyperthermia is the treatment of malignant tumors. MNPs embedded around a tumor site and placed under the action of an external oscillating magnetic field will heat up the local environment to a temperature of $41-46^{\circ} \mathrm{C}$, thus killing cancer cells [36]. For this treatment, magnetic fluids, that is, polydisperse colloidal solutions of single-domain MNPs (grains), are used. In aqueous system, MNPs tend to aggregate due to the long range Van der Waals' forces. Presence of a glycol layer on the surface of synthesized samples is beneficial for enhancing the dispersion and stabilizing the suspension.

The hyperthermia measurement was conducted for all samples simultaneously. Before presenting the results, some relevant theoretical considerations have to be mentioned. In an experiment performed over a long heating, the nonadiabatic processes due to heat loss released from the sample to surrounding environment should be taken into account. Such process could be described via change of temperature, $T$, over time, $t$ [37]:

$$
T(t)=T_{0}+\Delta T_{\max }\left(1-e^{-t / \tau}\right),
$$

where $T_{0}$ is the initial temperature (thermostat temperature) of the sample, $\Delta T_{\max }$ is the maximum change of the temperature in the stable state, and $\tau$ is the heating time constant.

SAR value can be calculated by the initial slope method, that is, by approximating the slope of the initial heat rise, $\beta$ :

$$
\beta=\left.\frac{d T}{d t}\right|_{t=0} .
$$

The underlying assumption is that the initial slope of the curve does not suffer from losses since the sample is at its initial temperature. Therefore, the SAR can be calculated from the following equation:

$$
\mathrm{SAR}=\frac{C m_{\mathrm{w}}}{m_{\text {sample }}} \beta,
$$

where $C$ is the heat capacity of the sample, $m_{\mathrm{w}}$ is the mass of the solvent (water), and $m_{\text {sample }}$ is the total mass of the sample in water [38].

The experimental results of the measured time changes in the temperature of magnetic fluids of $1 \mathrm{mg} / \mathrm{ml}$ and $2 \mathrm{mg} / \mathrm{ml}$ 

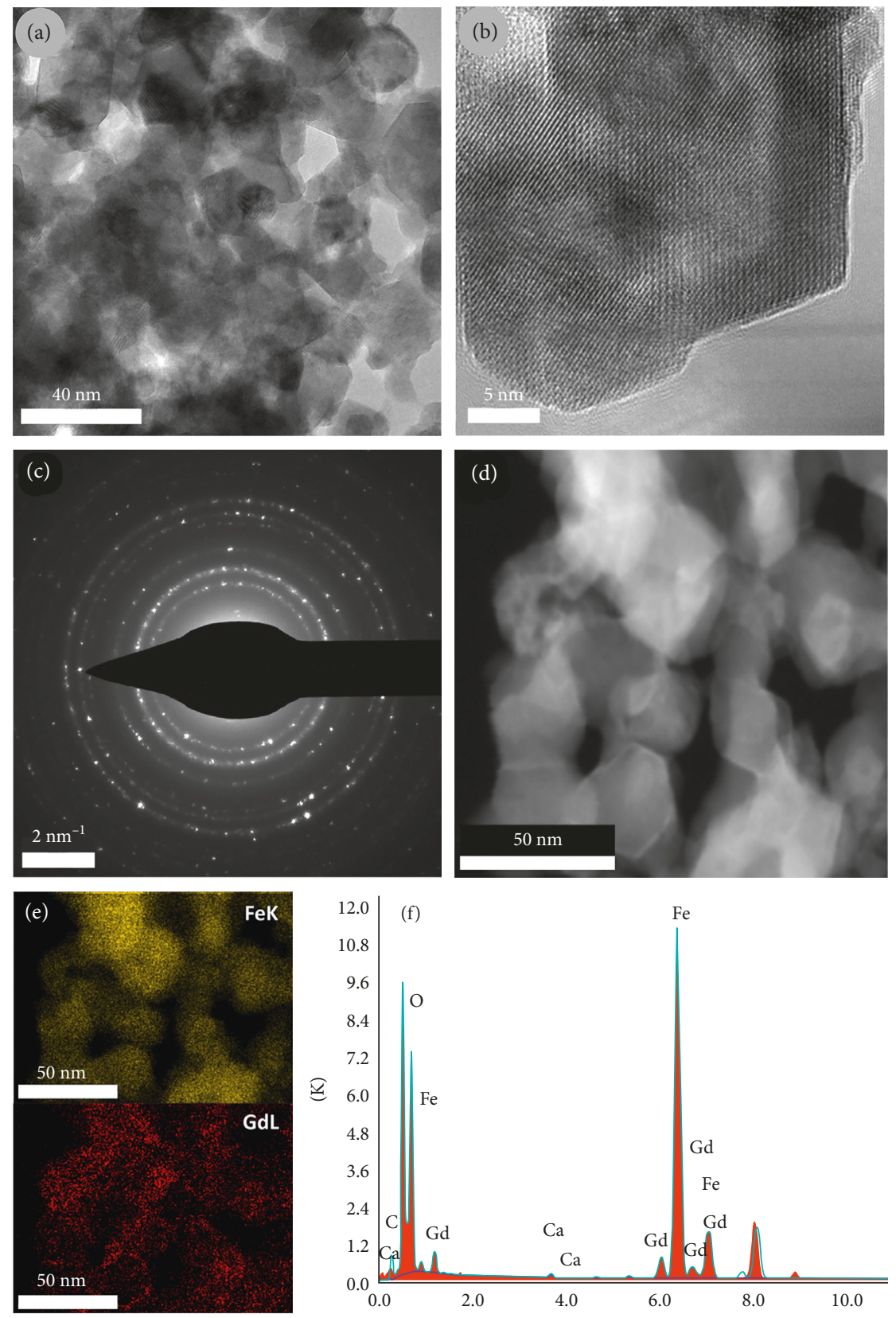

FIGURE 3: Electronic imaging of the MNP-Gd5.1\% sample: (a, b) HR-TEM images; (c) SAED image; (d) STEM image; (e) EDS mapping at the Fe K edge (above) and Gd L edge (below) on the selected area in image (d); (f) EDS histogram of elemental composition in a typical point of the sample.

concentrations under the action of the external magnetic field are presented in Figure 5, while calculated SAR values are summarized in Table 1. The results show that the synthesized materials demonstrate a good response to the oscillating magnetic field. Within 20-30 min of action, the water solution warms up by $3^{\circ} \mathrm{C}$. In case of performing hyperthermia on these fluids in vivo, this would allow to reach about $40-41^{\circ} \mathrm{C}$, a therapeutically desired level of temperature impact. There is a direct correlation between the concentration of MNPs in the probe and the final heating temperature. The obtained results are comparable with those described in [19], where MNPs having a ten times lower gadolinium content (from 0.02 to 0.08 mol.\%) were investigated.

\section{Conclusions}

The proposed MW-assisted polyol synthesis allows preparing $\mathrm{Gd}^{3+}$-doped MNPs in the 6-24 nm size range (TEM) with an average crystallite size of $14 \mathrm{~nm}$ (XRD). The actual values of $\mathrm{Gd}^{3+}$ doping of materials were in the range of $0.5-5.1 \mathrm{~mol}$ \% (XRF). Dopant is uniformly distributed in the volume of MNPs (STEM-EDS). The surface of NPs is 


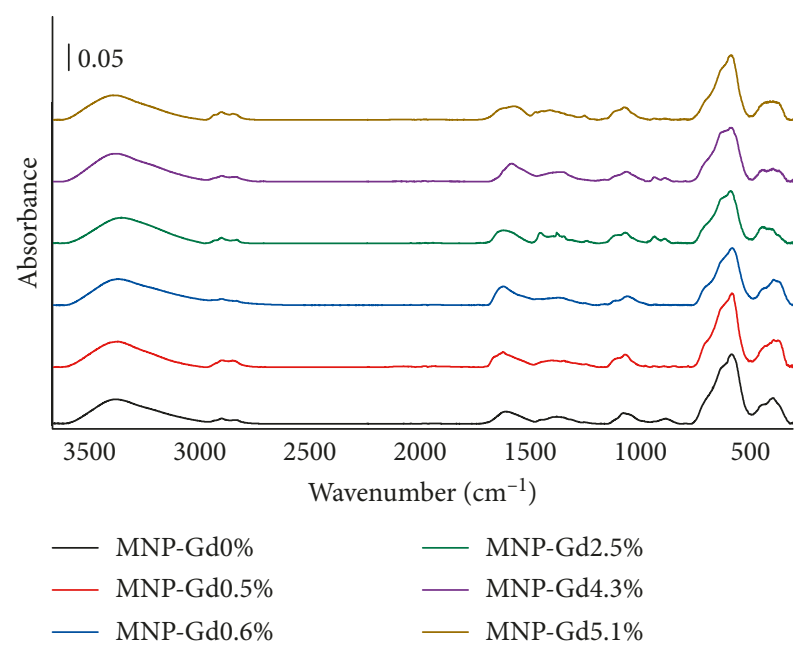

(a)

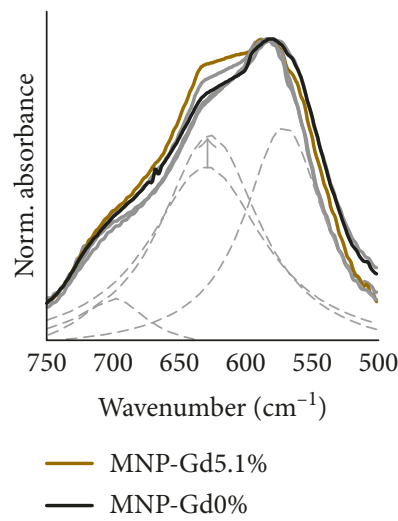

(b)

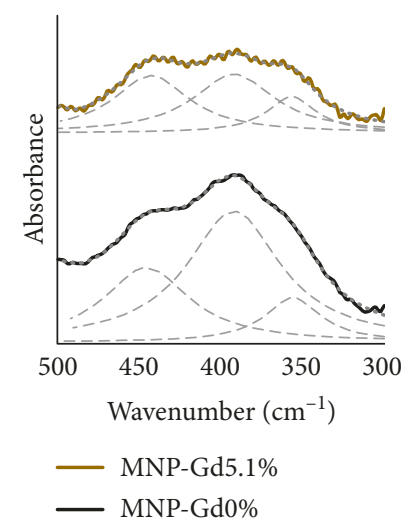

(c)

Figure 4: (a) FTIR spectra of synthesized samples: (1) MNP-Gd0\%; (2) MNP-Gd0.5\%; (3) MNP-Gd0.6\%; (4) MNP-Gd2.5\%; (5) MNPGd4.3\%; (6) MNP-Gd5.1\%. (b) An enlargement of the $500-750 \mathrm{~cm}^{-1}$ spectral interval reporting the absorption band centered at $\sim 600 \mathrm{~cm}^{-1}$ of normalized intensity together with fitting results for the MNP-Gd0\% sample. (c) An enlargement of $300-500 \mathrm{~cm}^{-1}$ spectral interval reporting the absorption band centered at $\sim 400 \mathrm{~cm}^{-1}$ for MNP-Gd0\% and MNP-Gd5.1\% samples with fitting results.



(a)

Figure 5: In the legends of figures 5a and 5b, please change (1), (2), (3), (4), (5), and (6) to MNP-Gd0\%, MNP-Gd0.5\%, MNP-Gd0.6\%, MNP-Gd2.5\%, MNP-Gd4.3\%, and MNP-Gd5.1\%, respectively.

covered by glycol molecules (FTIR). We also found that introduction of gadolinium (III) in quantities higher than $2.7 \mathrm{~mol} . \%$ alters the purity of a spinel phase of resulting MNPs. Hyperthermia test of 1 and $2 \mathrm{mg} / \mathrm{ml}$ aqueous colloidal solutions of MNPs demonstrated their heating ability at $3^{\circ} \mathrm{C}$ in $20-30 \mathrm{~min}$ run. These results confirm the relevance of synthesized materials for biomedical applications.

\section{Data Availability}

X-ray powder diffraction raw data are available in Supplementary Materials.

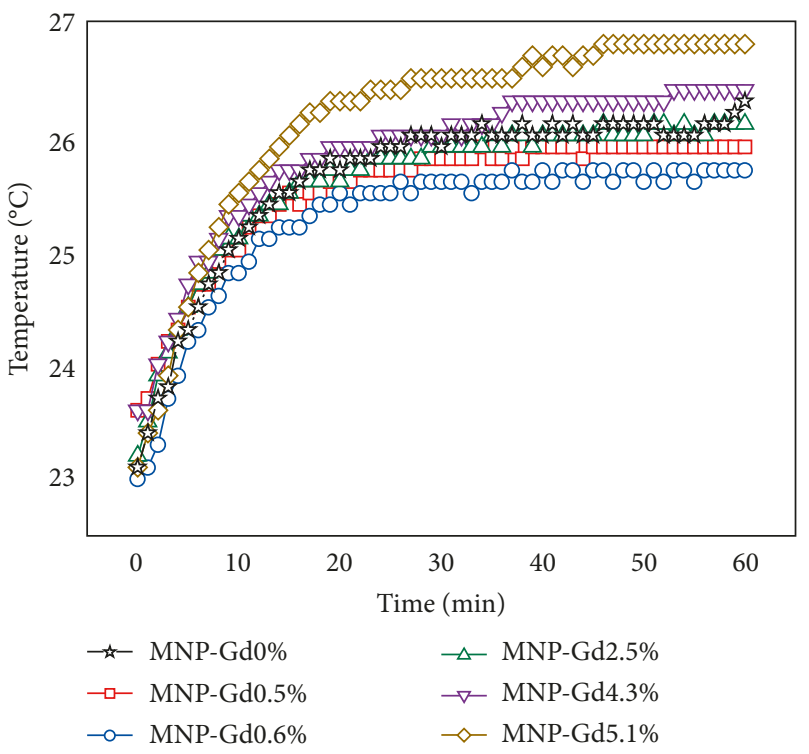

(b)

\section{Conflicts of Interest}

The authors declare that there are no conflicts of interest regarding the publication of this paper.

\section{Acknowledgments}

The authors acknowledge the Ministry of Education and Science of the Russian Federation (Agreement no. 14.587.21.0027, unique identifier RFMEFI58716X0027) for the financial support. The authors are indebted to Roman Matveev, SFedU, for performing the hyperthermia 
measurements and Tatiana Klochko for her support and assistance during the synthesis.

\section{Supplementary Materials}

Supplementary materials contain X-ray diffraction patterns in the XYE format, where the first column represents the $2 \theta$ values, second column is the scattered intensity, and the final column contains absolute error according to the Poisson statistics. (Supplementary Materials)

\section{References}

[1] W. Wu, Z. Wu, T. Yu, C. Jiang, and W.-S. Kim, "Recent progress on magnetic iron oxide nanoparticles: synthesis, surface functional strategies and biomedical applications," Science and Technology of Advanced Materials, vol. 16, no. 2, article 023501, 2015.

[2] R. Easterday, O. Sanchez-Felix, Y. Losovyj et al., "Design of ruthenium/iron oxide nanoparticle mixtures for hydrogenation of nitrobenzene," Catalysis Science and Technology, vol. 5, no. 3, pp. 1902-1910, 2015.

[3] Y. Tuo, G. Liu, B. Dong et al., "Microbial synthesis of $\mathrm{Pd} /$ $\mathrm{Fe}_{3} \mathrm{O}_{4}, \mathrm{Au} / \mathrm{Fe}_{3} \mathrm{O}_{4}$ and $\mathrm{PdAu} / \mathrm{Fe}_{3} \mathrm{O}_{4}$ nanocomposites for catalytic reduction of nitroaromatic compounds," Scientific Reports, vol. 5, no. 1, pp. 13515-13527, 2015.

[4] Q. A. Pankhurst, J. Connolly, S. K. Jones, and J. Dobson, "Applications of magnetic nanoparticles in biomedicine," Journal of Physics D: Applied Physics, vol. 36, no. 13, pp. R167-R181, 2003.

[5] Y. Bagbi, A. Sarswat, D. Mohan, A. Pandey, and P. R. Solanki, "Lead and chromium adsorption from water using L-cysteine functionalized magnetite $\left(\mathrm{Fe}_{3} \mathrm{O}_{4}\right)$ nanoparticles," Scientific Reports, vol. 7, no. 1, pp. 7672-7687, 2017.

[6] M. Auffan, J. Achouak, J. Rose et al., "Relation between the redox state of iron-based nanoparticles and their cytotoxicity toward Escherichia coli," Environmental Science \& Technology, vol. 42, no. 17, pp. 6730-6735, 2008.

[7] J. A. R. Guivar, E. Sadrollahi, D. Menzel et al., "Magnetic, structural and surface properties of functionalized maghemitenanoparticles for copper and lead adsorption," RSC Advances, vol. 7, no. 46, pp. 28763-28779, 2017.

[8] S. Sun, C. B. Murray, D. Weller, L. Folks, and A. Moser, "Monodisperse FePt nanoparticles and ferromagnetic FePt nanocrystal superlattices," Science, vol. 287, no. 5460, pp. 1989-1992, 2000.

[9] E. Brok, J. Larsen, M. Varón, W. T. Hansen, and C. Frandsen, "Induced mesocrystal-formation, hydrothermal growth and magnetic properties of $\alpha-\mathrm{Fe}_{2} \mathrm{O}_{3}$ nanoparticles in salt-rich aqueous solutions," Crystals, vol. 7, no. 8, p. 248, 2017.

[10] S. Lentijo Mozo, E. Zuddas, A. Casu, and A. Falqui, "Synthesizing iron oxide nanostructures: the polyethylenenemine (PEI) role," Crystals, vol. 7, no. 1, p. 22, 2017.

[11] F. Ooi, J. S. DuChene, J. Qiu et al., "A facile solvothermal synthesis of octahedral $\mathrm{Fe}_{3} \mathrm{O}_{4}$ nanoparticles," Small, vol. 11, no. 22, pp. 2649-2653, 2015.

[12] S. P. Gubin, "Introduction," in Magnetic Nanoparticles, pp. 1-23, Wiley-VCH Verlag GmbH and Co., KGaA, Weinheim, Germany, 2009.

[13] G. B. Deacon and R. J. Phillips, "Relationships between the carbon-oxygen stretching frequencies of carboxylato complexes and the type of carboxylate coordination,"
Coordination Chemistry Reviews, vol. 33, no. 3, pp. 227-250, 1980.

[14] J. Roger, J. N. Pons, R. Massart, A. Halbreich, and J. C. Bacri, "Some biomedical applications of ferro fluids," European Physical Journal Applied Physics, vol. 5, no. 3, pp. 321-325, 1999.

[15] R. Jurgons, C. Seliger, A. Hilpert, L. Trahms, S. Odenbach, and C. Alexiou, "Drug loaded magnetic nanoparticles for cancer therapy," Journal of Physics: Condensed Matter, vol. 18, no. 38, pp. S2893-S2902, 2006.

[16] C. Sun, J. Lee, and M. Zhang, "Magnetic nanoparticles in MR imaging and drug delivery," Advanced Drug Delivery Reviews, vol. 60, no. 11, pp. 1252-1265, 2008.

[17] Z. P. Xu, Q. H. Zeng, G. Q. Lu, and A. B. Yu, "Inorganic nanoparticles as carriers for efficient cellular delivery," Chemical Engineering Science, vol. 61, no. 3, pp. 1027-1040, 2006.

[18] H. Lee, H. P. Shao, Y. Q. Huang, and B. Kwak, "Synthesis of MRI contrast agent by coating superparamagnetic iron oxide with chitosan," IEEE Transactions on Magnetics, vol. 41, no. 10, pp. 4102-4104, 2005.

[19] N. D. Thorat, R. A. Bohara, H. M. Yadav, and T. Syed, "Multimodal MR imaging and magnetic hyperthermia study of Gd doped $\mathrm{Fe}_{3} \mathrm{O}_{4}$ nanoparticles for integrative cancer therapy," RSC Advances, vol. 6, no. 97, pp. 94967-94975, 2016.

[20] C. R. De Silva, S. Smith, I. Shim et al., "Lanthanide(III)-doped magnetite nanoparticles," Journal of the American Chemical Society, vol. 131, no. 18, pp. 6336-6337, 2009.

[21] W. Huan, C. Cheng, Y. Yang, H. Yuan, and Y. Li, "A study on the magnetic and photoluminescence properties of $\mathrm{Eu}^{\mathrm{n}+}$ and $\mathrm{Sm}^{3+}$ doped $\mathrm{Fe}_{3} \mathrm{O}_{4}$ nanoparticles," Journal of Nanoscience and Nanotechnology, vol. 12, no. 6, pp. 4621-4634, 2012.

[22] E. S. Choi, W. Xu, M. J. Baek et al., "Gd(III) doping effect on magnetization and water proton relaxivities in ultra small iron oxide nanoparticles," AIP Advances, vol. 3, no. 7, article 072101, 2013.

[23] T. A. Lastovina, S. A. Efimova, E. A. Kudryavtsev, and A. V. Soldatov, "Preparation of the $\mathrm{Sm}^{3+}$-doped magnetic nanoparticles via microwave-assisted polyol synthesis," Bionanoscience, vol. 7, no. 1, pp. 4-10, 2016.

[24] T. A. Lastovina, A. P. Budnyk, E. A. Kudryavtsev et al., "Solvothermal synthesis of $\mathrm{Sm}^{3+}$-doped $\mathrm{Fe}_{3} \mathrm{O}_{4}$ nanoparticles," Materials Science and Engineering: C, vol. 80, pp. 110-116, 2017.

[25] T. A. Lastovina, A. L. Bugaev, S. P. Kubrin, E. A. Kudryavtsev, and A. V. Soldatov, "Structural studies of magnetic nanoparticles doped with rare-earth elements," Journal of Structural Chemistry, vol. 57, no. 7, pp. 1444-1449, 2017.

[26] E. Aivazoglou, E. Metaxa, and E. Hristoforou, "Microwaveassisted synthesis of iron oxide nanoparticles in biocompatible organic environment," AIP Advances, vol. 8, no. 4, article 048201, 2018.

[27] V. Petř́iček, M. Dušek, and L. Palatinus, "Crystallographic computing system JANA2006: general features," Zeitschrift für Kristallographie, vol. 229, no. 5, pp. 345-352, 2014.

[28] A. L. Bugaev, V. A. Polyakov, A. A. Tereshchenko et al., "Chemical synthesis and characterization of $\mathrm{Pd} / \mathrm{SiO}_{2}$ : the effect of chemical reagent," Metals, vol. 8, no. 2, p. 135, 2018.

[29] A. L. Bugaev, A. A. Guda, K. A. Lomachenko et al., "Coreshell structure of palladium hydride nanoparticles revealed by combined X-ray absorption spectroscopy and X-ray diffraction," Journal of Physical Chemistry C, vol. 121, no. 33, pp. 18202-18213, 2017. 
[30] Z. Kozakova, I. Kuritka, N. E. Kazantseva et al., "The formation mechanism of iron oxide nanoparticles within the microwave-assisted solvothermal synthesis and its correlation with the structural and magnetic properties," Dalton Transactions, vol. 44, no. 48, pp. 21099-21108, 2015.

[31] H. C. Liese, "An infrared absorption analysis of magnetite," American Mineralogist, vol. 52, pp. 1198-1205, 1967.

[32] M. Ishii and M. Nakahira, "Infrared absorption spectra and cation distribution in $(\mathrm{Mn}, \mathrm{Fe})_{3} \mathrm{O}_{4}$," Solid State Communications, vol. 11, no. 1, pp. 209-212, 1972.

[33] H. Namduri and S. Nasrazadani, "Quantitative analysis of iron oxides using Fourier transform infrared spectrophotometry," Corrosion Science, vol. 50, no. 9, pp. 2493-2497, 2008.

[34] H. Frei, T.-K. Ha, R. Meyer, and H. H. Gunthard, "Ethylene glycol: infrared spectra, ab initio calculations, vibrational analysis and conformations of 5 matrix isolated isotopic modifications," Chemical Physics, vol. 25, no. 2, pp. 271-298, 1977.

[35] B. Thapa, D. Diaz-Diestra, J. Beltran-Huarac, B. R. Weiner, and G. Morell, "Enhanced MRI T2 relaxivity in contrast probed anchor-free PEGylated iron oxide nanoparticles," Nanoscale Research Letters, vol. 12, no. 1, p. 312, 2017.

[36] A. K. Gupta and M. Gupta, "Synthesis and surface engineering of iron oxide nanoparticles for biomedical applications," Biomaterials, vol. 26, no. 18, pp. 3995-4021, 2005.

[37] A. Skumiel, "Suitability of water based magnetic fluid with $\mathrm{CoFe}_{2} \mathrm{O}_{4}$ particles in hyperthermia," Journal of Magnetism and Magnetic Materials, vol. 307, no. 1, pp. 85-90, 2006.

[38] R. R. Wildeboer, P. Southern, and Q. A. Pankhurst, "On the reliable measurement of specific absorption rates and intrinsic loss parameters in magnetic hyperthermia materials," Journal of Physics D: Applied Physics, vol. 47, no. 49, pp. 495003495017, 2014. 

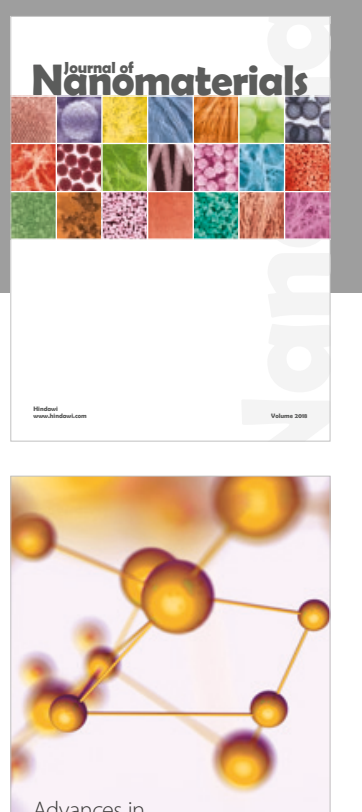

Physical Chemistry
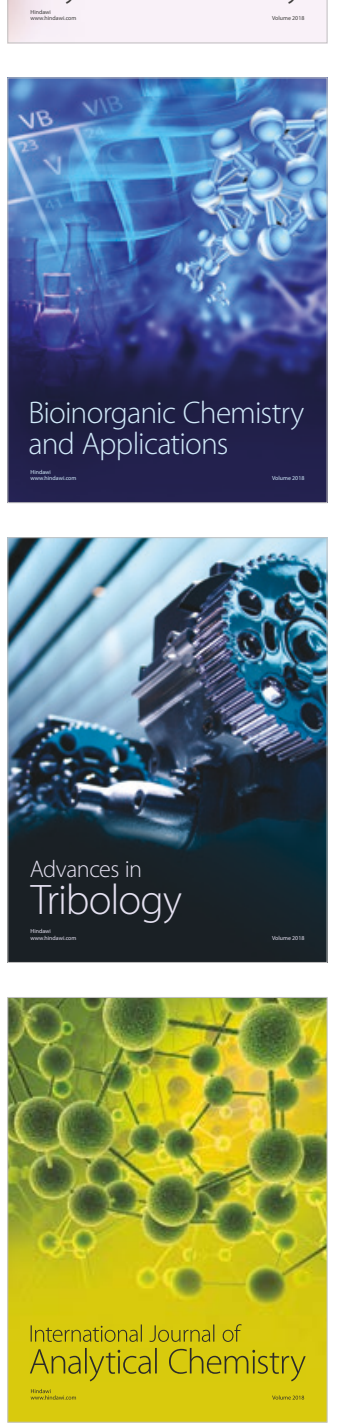

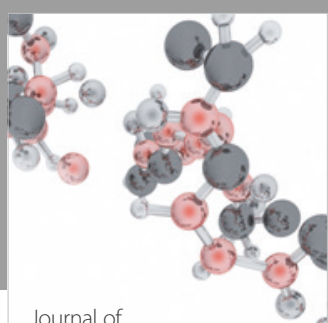

Analytical Methods

in Chemistry

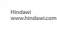

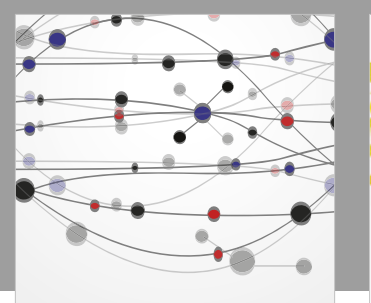

The Scientific World Journal

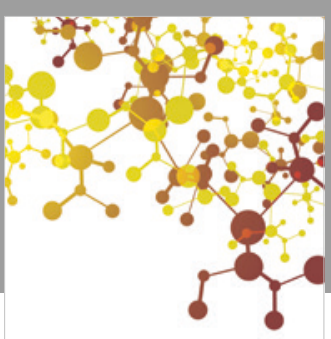

Journal of

Applied Chemistry


Submit your manuscripts at

www.hindawi.com
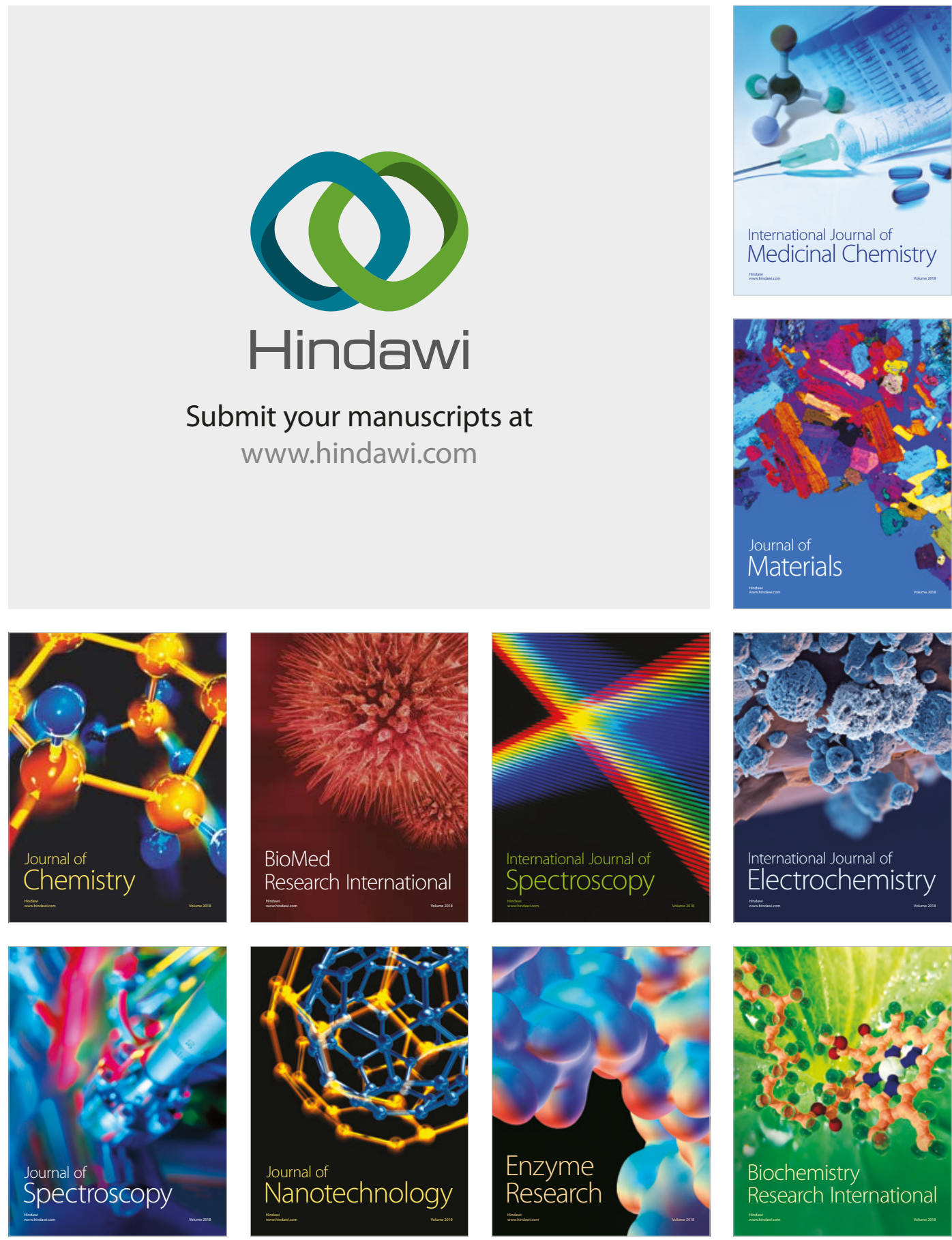
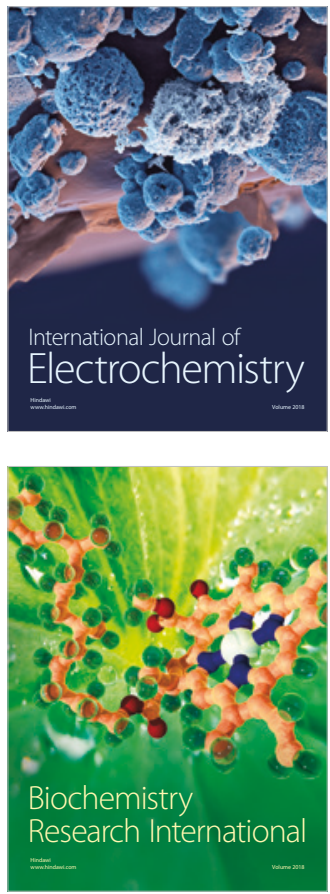